\title{
Single Neuronal and Glial Gene Expression in the Nucleus Tractus Solitarius in an Alcohol Withdrawal Time Series Reveals Novel Cellular Phenotypes and Networks
}

\author{
Sean J. O'Sullivan \\ Thomas Jefferson University \\ Damani McIntosh -Clarke \\ Thomas Jefferson University \\ James Park \\ Thomas Jefferson University \\ Rajanikanth Vadigepalli \\ Thomas Jefferson University \\ James S . Schwaber ( $\nabla$ james.schwaber@jefferson.edu ) \\ Thomas Jefferson University
}

\section{Research Article}

Keywords: Alcohol withdrawal syndrome (AWS), neuronal hyperexcitability, autonomic dysregulation, nucleus tractus solitarius (NTS)

Posted Date: May 6th, 2021

DOl: https://doi.org/10.21203/rs.3.rs-452767/v1

License: (c) (i) This work is licensed under a Creative Commons Attribution 4.0 International License.

Read Full License 


\section{Abstract}

Alcohol withdrawal syndrome (AWS) is characterized by neuronal hyperexcitability, autonomic dysregulation, and severe negative emotion. The nucleus tractus solitarius (NTS) is largely responsible for the neurological processes underlying these symptoms as it is the main viscerosensory nucleus in the brain. The NTS receives visceral interoceptive inputs, influences autonomic outputs, and has strong connections to the limbic system and hypothalamic-pituitary-adrenal axis to maintain homeostasis. Our prior analysis of single neuronal gene expression data from the NTS shows that neurons exist in heterogeneous transcriptional states that form distinct functional subphenotypes. Our working model conjectures that chronic ethanol induces a state of allostasis in which NTS neurons and peripheral tissues generally compensate for the persistent presence of alcohol, and that abrupt abstinence causes central and peripheral biological network decompensation resulting in the observed AWS. We collected single noradrenergic and glucagon-like peptide-1 (GLP-1) neurons and microglia from rat NTS and measured a subset of their transcriptome in an alcohol withdrawal time series. Inflammatory subphenotypes predominate at certain time points, and GLP-1 subphenotypes demonstrated hyperexcitability post-withdrawal. We hypothesize such inflammatory and anxiogenic signaling contributes to alcohol dependence via negative reinforcement. Targets to mitigate such dysregulation and treat dependence can be identified from this dataset.

\section{Introduction}

Alcohol withdrawal syndrome (AWS) is characterized by negative physical and emotional symptoms. Physical symptoms are driven by autonomic dysregulation, $y$-aminobutyric acid (GABA) hypoactivity, and increased glutamatergic signaling leading to dysphoria, nausea, diaphoresis, tachycardia, hypertension, seizures, and delirium tremens ${ }^{1}$. Fear and anxiety are the principle emotional symptoms. The negative reinforcement hypothesis of substance dependence postulates that these negative physical and emotional symptoms experienced in withdrawal motivate alcohol dependence ${ }^{2-5}$. We conjecture that peripheral network decompensation is a central facet of this model, and that neurovisceral feedback via the vagus nerve conveying peripheral information to the central nervous system contributes substantially to the severity of symptoms experienced ${ }^{6}$ (Fig. 1A-B). Investigation into the underlying mechanisms producing these symptoms may provide insight into targets that mitigate acute and protracted AWS severity and prevent relapse following abstinence. Such treatments may provide clinical utility for other substances of abuse with severe withdrawal syndromes such as opioids.

Neuroinflammatory processes have emerged as an important contributor to the severity of AWS symptoms, especially in the amygdala ${ }^{6-14}$. The amygdala is strongly implicated in threat detection and negative emotion, and inflammation here may drive some of the fear and anxiety experienced in $\mathrm{AWS}^{6,15,16}$. The likely mechanism underlying this phenomenon is that inflammation causes neuronal hyperexcitability ${ }^{17}$. 
The nucleus tractus solitarius (NTS) is another brain region that contributes to the symptoms of AWS and is implicated in alcohol dependence ${ }^{18-22}$. The NTS receives visceral inputs from the interoceptive vagal circuit, strongly influences autonomic outputs, and has strong bidirectional connections to the amygdala (Fig. 1A). Connections to the paraventricular nucleus, ventrolateral medulla, and amygdala place the NTS in the center of the visceral-emotional neuraxis (Fig. 1B) ${ }^{6,23,24}$. Many of these connections use the neuropeptide glucagon-like peptide-1 (GLP-1) as a transmitter. Indeed, interoceptive vagal afferents synapse onto GLP-1 positive (+) NTS neurons that go on to form anxiogenic synapses ${ }^{25-29}$. Recently, GLP-1R activity in the NTS has been linked to alcohol-mediated behavior ${ }^{30}$. Additionally, the NTS houses noradrenergic (NE) neurons that also respond to vagal and higher-order inputs and principally function to maintain cardiovascular homeostasis (Fig. 1B). These NE + neurons also project to the amygdala where they contribute to emotional memory $24,31-33$. We conjecture a model in which GLP-1 and NE neurotransmission form parallel complementary pathways conveying the peripheral state via NTS to the limbic forebrain (Fig. 1A-B).

Given their function in autonomic homeostasis and emotion, NE + and GLP-1 + neurons in the NTS likely contribute to the negative physical and emotional symptoms of AWS and negative reinforcement motivating alcohol dependence in overlapping and distinct ways. Further, inflammatory glial-neuronal signaling in the NTS during AWS may also contribute to the severity of physical and emotional withdrawal symptoms ${ }^{6,9,10}$. Local inflammatory signaling in the NTS contributes to the development of hypertension in rats implicating paracrine cytokine involvement in $\mathrm{AWS}^{34,35}$. Moreover, the antineuroinflammatory molecule ibudilast is in clinical trials to reduce alcohol craving and AWS severity ${ }^{36}$.

Here, we measured how the functional states of single NE neurons, GLP-1 neurons, and microglia in the NTS change over the course of alcohol withdrawal. Single-cell approaches allow for the identification of cellular subphenotypes-morphologically indistinguishable cells anatomically localized that use the same primary neurotransmitter yet have distinct transcriptomic profiles. Our previous work has demonstrated the heterogeneity of single-cells, and the functional importance of subphenotypes that may be missed in tissue-level approaches ${ }^{37,38}$. Changes in transcription during alcohol withdrawal revealed a pattern suggesting peak dysregulation and inflammation at the 32 hour (hr) withdrawal (wd) time point. Additionally, the expression of $\mathrm{GABA}_{\mathrm{A}}$ receptor $(\mathrm{R})$ subunits genes was downregulated in protracted withdrawal, measured as the $176 \mathrm{hr}$ wd time point, suggesting hyperexcitability of anxiogenic GLP-1 + neurons.

\section{Results}

We used laser capture microdissection (LCM) to gather single cells from rat NTS in control, chronic ethanol (EtOH), 8 hour (hr) withdrawal (wd), $32 \mathrm{hr} w d$, or $176 \mathrm{hr}$ wd treatments (Sup. Figure 1A). We subsequently used microfluidic RT-qPCR to measure a subset of the transcriptome in these single-cells (Sup. Figure 1B) ${ }^{39}$. Following strict quality control protocols, a total of 229 10-cell pooled samples (700 NE neurons, 650 GLP-1 neurons, and 940 microglia) and 65 gene transcripts were used for data analysis 
(Fig. 1C, Sup. Table 1-2). We targeted gene transcripts involved in inflammatory glial-neuronal signaling and $G_{A B A} R$ subunits. Cellular phenotype selection was validated by the expression of the cell type markers NeuN, Cd34, and Cx3cr7 (Fig. 1D). Neurons were selected based on NEUN and TH immunofluorescence. TH-neurons demonstrated significantly elevated levels of the Gcg transcript that codes for the GLP-1 precursor protein suggesting these samples constitute GLP-1 enriched neurons (Fig. 1D). A dimensionality reduction analysis (linear discriminate analysis) further demonstrated the differences between the three cell types gathered. Microglia differed from neurons along the $x$-axis, NE neurons and GLP-1 enriched neurons differed from each other along the y-axis (Fig. 1E).

The expression of the neurotransmitter precursor genes Th and Gcg for NE and GLP-1 neurons, respectively, is plotted across treatment time points in Fig. 2A and B. We find that expression of these transcripts is inversely correlated-at time points in which Th expression is relatively high in NE neurons, Gcg expression is relatively low in GLP-1 neurons, and vice versa (Fig. 2A-B). This suggests a push-pull dynamic mechanism in the genetic regulation of neurotransmission by these neurons. Notably, Gcg expression was induced in the three withdrawal time points, but suppressed in control and $\mathrm{EtOH}$ treatments suggesting GLP-1 neurotransmission is pathologically elevated during the withdrawal process. At the $176 \mathrm{hr}$ wd time point, bimodal distribution of Th expression in NE neurons and trimodal Gcg expression in GLP-1 neurons is observed (Fig. 2A-B). High and low Th-expressing NE neurons and Gcg-expressing GLP-1 neurons from this time point were separated into heat maps organized by Euclidian distance clustering of gene expression (Fig. 2C-D). Th expression in NE neurons and Gcg expression in GLP-1 neurons is moderately predictive of cellular subphenotypes that loosely organize coexpression gene clusters. However, Th-expression and Gcg-expression alone, which is to say neurotransmitter expression, is not the best determinate of cellular subsphenotypes-a finding we have observed previously in other neuronal nuclei ${ }^{38}$.

Heat maps establishing well-defined data-driven cellular subphenotypes in neurons and microglia were generated using Euclidean distance clustering of z-scores of the $-\Delta \Delta \mathrm{C}_{\mathrm{t}}$ values for each sample and gene in the dataset (Figs. 3,4,5). Co-expression gene clusters are labeled with numbers and cellular subphenotype groupings are labeled with letters. GLP-1 neurons had the same cellular subphenotypes with the same gene clusters across all treatments while NE neurons and microglia had two co-expression configurations comprising the identified subphenotypes. The proportion of cells constituting a subphenotype in addition to gene cluster expression levels shifted with the treatment. GABA $R$ subunit genes clustered together in every configuration, and their expression was largely indicative of subphenotype groupings.

Two prominent subphenotypes, $C$ and $D$, emerged in NE neurons in withdrawal time points. Subphenotype $C$ highly expressed gene cluster 4 which is rich in inflammatory ligands and receptors including $\mathrm{Crh}, / / 1 \mathrm{~b}$, and Ptgs2. This was labeled the 'inflammatory' gene cluster (see below). Subphenotype $C$ suppressed gene cluster 5 , which includes $T h$ and $G_{A B A} R$ subunits, and 6 . Subphenotype $\mathrm{D}$, conversely, had the opposite expression pattern in these gene clusters. At $8 \mathrm{hr}$ wd, 
subphenotype $\mathrm{C}$ was predominant, but subphenotype $\mathrm{D}$ made up a higher proportion of cells at 32 and $176 \mathrm{hr}$ wd. Another subphenotype, E, emerged in the $32 \mathrm{hr}$ wd treatment that had moderately high expression of gene clusters 4 and 5 . Subphenotypes D and E were further split into D1, D2, E1, and E2 based on medium or high expression, respectively, of gene cluster 6 which includes Cd200, cFos, and Mif.

$\mathrm{NE}$ neurons from control and $\mathrm{EtOH}$ treatments shared gene clusters distinct from the withdrawal treatments. $G A B A_{A} R$ subunits were co-expressed consistent with all sampled cell types. $G A B A_{A} R$ subunit genes showed elevated expression in subphenotype $\mathrm{B}$ in control and $\mathrm{EtOH}$ treatments, moderate expression in subphenotype $\mathrm{D}$ and $\mathrm{E}$ in 8 and $32 \mathrm{hr}$ wd treatments, and returned to high expression in 176 $\mathrm{hr}$ wd subphenotype $\mathrm{D}$. In subphenotype $\mathrm{E}$, only found at $32 \mathrm{hr}$ wd, all assayed genes were at least moderately expressed suggesting the regulatory expression mechanisms that control this transcriptomic profile are stimulated to this phase of the withdrawal process.

Co-expression genes clusters in GLP-1 neurons were consistent throughout the time series. Subphenotype A highly expressed 'inflammatory' gene cluster 1 rich in cytokine and chemokine ligands and receptors including $C r h, / / 1 b$, and Ptgs2, while suppressing 'GABA $A_{A} R^{\prime}$ gene cluster 2 . Subphenotype $B$ had the opposite pattern. GLP-1 co-expression clusters 1 and 2 were surprisingly similar to NE co-expression clusters 4 and 5 , again suggesting the mechanisms of regulatory constraint are shared between these phenotypes. Interestingly, GLP-1 subphenotype B emerged only in the EtOH treatment. At $8 \mathrm{hr} w d$, subphenotypes $A$ and $B$ suppressed expression of their high-expressing gene clusters, 1 and 2 respectively, compared to control. At 32 and $176 \mathrm{hr}$ wd, subphenotype A gene cluster 1 was more highly expressed than in the control condition. Subphenotype $B$ gene demonstrated a steady decrease in expression of gene cluster 2 , and by the $176 \mathrm{hr}$ wd time had only moderate expression of 'GABA $R_{A}$ ' gene cluster 2. Concurrently, expression of gene cluster 3 for subphenotype $B$ consistently increases throughout the withdrawal process and by $176 \mathrm{hr}$ wd is the most prominently upregulated gene cluster. Tnf did not group into any gene cluster and is isolated in GLP-1 neuronal heat map to display this clearly (Fig. 4).

Microglia shared co-expression clusters in subphenotypes A, B, and C for control, $32 \mathrm{hr}$ wd, and $176 \mathrm{hr}$ wd treatments. EtOH and $8 \mathrm{hr}$ wd treatments shared co-expression clusters in subphenotypes $\mathrm{D}$ and $\mathrm{E}$. Subphenotype A was the exclusive expression pattern for the $32 \mathrm{hr}$ wd treatment which upregulated 'inflammatory' gene cluster 1 including $C r h, / / 1 b$, and Ptgs2. This subphenotype A is most similar to the so-called M1 phenotype ${ }^{40}$. At $176 \mathrm{hr}$ wd, subphenotype A made up a low proportion of samples while subphenotype $\mathrm{C}$ moderately expressed all genes assayed and more robustly than in the control treatment. Tnf did not group into a gene cluster for microglia either. High expression of $G_{A B A} R$ Rs and a few other genes including Sod1, Cd200, Mapk1, and Stat3 characterized Subphenotype E in the EtOH and $8 \mathrm{hr}$ wd treatments.

These data are further displayed in cellular diagrams in which expression for each gene in the corresponding subphenoytpe is designated by a box that is colored by a scale represent the average zscore of $-\Delta \Delta \mathrm{C}_{t}$ expression values (Figs. 6,7,8). The location of this box in the cellular cartoon 
corresponds to the protein function of that gene. These diagrams provide a higher-level display of the functional state of the subphenotype at that time point. Many dynamic processes can be observed in these figures. In brief, Fig. 6 shows a clear upregulation of $G_{A B A} R$ genes at $176 \mathrm{hr}$ wd as compared to 8 $\mathrm{hr}$ wd in Group C and D NE neurons. Additionally, CD200 expression is one of the primary distinguishers of Group D1 vs. GroupD2. Figure 7 displaying GLP-1 neurons shows that GABA $A$ R gene expression at 176 $\mathrm{hr}$ wd is decreased in both subphenotypes. At the $32 \mathrm{hr}$ wd time point, $\mathrm{Cxc} / 10, \mathrm{Cxcr1}, \mathrm{Cxcr} 2$, and $\mathrm{Cxcr} 3$ expression distinguish Group A1 most prominently from Group A2. Microglia displayed in Fig. 8 showed the most Tnf expression in groups $\mathrm{D}$ and $\mathrm{E}$, subphenotypes only identified at the $\mathrm{EtOH}$ and $8 \mathrm{hr}$ wd treatments. $C \times 3 \mathrm{Cr} 1$, a mircoglia gene prominently involved in neuronal adhesion, showed increased expression at the $176 \mathrm{hr}$ wd time point in all subphenotypes ${ }^{41}$. GLP-1 Group B neurons had the most increased $\mathrm{C} \times 3 \mathrm{c} / 1$ expression suggesting this subphenotype interacts most with microglia at this time point.

\section{Discussion}

NTS neurons regulate emotion, autonomic homeostasis, and stress responses. Multiple neuronal nuclei, ligands, receptors, and signaling dynamics are involved in these complex functions including NE, GLP-1, $\mathrm{CRH}$, and GABA (Fig. 1A-B). Moreover, local glial-neuronal paracrine signaling via inflammatory cytokines like tumor necrosis factor-alpha (TNF-a) also play a role. We microdissected single $T h+$ neurons, Thneurons, and microglia from the rat NTS as 10-cell pooled samples using LCM and measured their expression of 96 gene transcripts in an alcohol withdrawal time series (Sup. Figure 1). Time points were chosen based on rat alcohol metabolism ${ }^{42} .8 \mathrm{hr}$ wd represents the start of acute AWS, $32 \mathrm{hr}$ wd represents the end of acute AWS, and $176 \mathrm{hr}$ wd represents a protracted withdrawal state. We found that neurons that stained $\mathrm{Th}+$ had significantly elevated Th expression and labeled them NE neurons (Fig. 1D). Th- neurons had significantly elevated expression of the GLP-1 precursor transcript Gcg, and were labeled as neuronal samples enriched with GLP-1 + neurons. Likewise, CD11 $\beta$ + cells expressed the microglial markers $C d 34$ and $C \times 3 c r 1$ at significantly elevated levels and were labeled microglia. In a dimension reduction analysis (LDA), these three cell types formed distinct clusters with microglia separating out from neurons along the $x$-axis and NE and GLP-1 neurons separating along the $y$-axis (Fig. 1E).

Further analysis of Th and Gcg expression showed an inverse relationship with respect to time point with Gcg expression demonstrating elevated expression levels only during withdrawal (Fig. 2A-B). However, expression of these neurotransmitter precursor genes did not organize the other genes assayed into distinct subphenotypes correlated to their expression levels (Fig. 2C-D). A data-driven approach to cellular subphenotype organization identified stark subphenotypes unique to each cell type likely with discrete functions (Figs. 3-5). Strikingly, these subphenotypes shared similarities in their expression of their inflammatory gene clusters (Sup. Table 4).

Gene cluster 4 in NE neurons, gene cluster 1 in GLP-1 neurons, and gene cluster 1 in microglia constituted these 'inflammatory' clusters (Sup. Table 4). 18 genes were shared across all of these co-expression 
clusters and only 5 genes were unique to a single cluster suggesting similar mechanisms across cell types that regulate their expression. In NE neurons, subphenotype $\mathrm{C}$ highly expressed this inflammatory cluster while subphenotype $\mathrm{E}$ had moderate inflammatory co-expression cluster elevation. At $8 \mathrm{hr} w \mathrm{w}, \mathrm{NE}$ subphenotype $C$ was $62.5 \%$ of the samples (5/8) and at $32 \mathrm{hr}$ wd $C$ and $E$ combined to $62.2 \%$ of the samples (23/37). By $176 \mathrm{hr}$ wd, subphenotype C was only $29 \%$ of NE neuron samples (5/17). This suggests that this subphenotype of NE neurons experiences a marked increase in inflammation during acute AWS, but that this subphenotype is not involved in protracted withdrawal symptoms such as lowgrade anxiety ${ }^{12}$. This increase in local paracrine inflammation likely increases the excitability for this NE neuron subphenotype ${ }^{17}$. This is consistent with the observation of hypersympathetic activity in acute, but not prolonged, $\mathrm{AWS}^{1}$.

In GLP-1 neurons, subphenotype A highly expressed the 'inflammatory' gene cluster (gene cluster 1 ). The pattern of expression in this inflammatory subphenotype of GLP-1 neurons (A) is similar to the NE inflammatory subphenotypes ( $C$ and $E$ ). GLP-1 Subphenotype A makes up $33.3 \%$ of control samples (3/9) and $0 \%$ of EtOH samples. At $8 \mathrm{hr} w d, 62.5 \%$ (5/8) of GLP-1 neurons are inflammatory subphenotype $A$ and at $32 \mathrm{hr}$ wd it is $55.2 \%(16 / 29)$. By $176 \mathrm{hr} w d$, this inflammatory subphenotype has decreased back near control levels: $35.7 \%(5 / 14)$ of GLP-1 neurons.

Surprisingly, microglia demonstrated a similar pattern. Microglia subphenotype A also highly expressed the inflammatory gene cluster (cluster 1). High gene expression in this cluster is indicative of M1 microglia phenotypes as this cluster includes the M1 markers $\|1 \beta\|$,6 , Nos 1 , Ptgs 2 , and TLRs 1,4 , and $5^{43}$. This phenotype made up $29.0 \%$ (9/31) of the control samples, $100 \%(13 / 13)$ of the $32 \mathrm{hr}$ wd samples, and only $21.4 \%$ of the $176 \mathrm{hr}$ wd samples. Of note, all NTS microglia sampled at the $32 \mathrm{hr}$ wd time points demonstrated an M1 phenotype suggesting the importance of neuroinflammation in the NTS during the acute phase of AWS. Conversely, we observed fewer M1-like microglia at $176 \mathrm{hr}$ wd compared to control samples which is unexpected based on our previous work on alcohol withdrawal in the amygdala9,44,45. We expected neuroinflammatory markers to be increased at the $176 \mathrm{hr}$ wd time point, especially in microglia, but these data suggest that the NTS experiences inflammation during acute withdrawal only (8 $\mathrm{hr}$ and $32 \mathrm{hr}$ time points) and recovers by the $176 \mathrm{hr}$ time point. We observe this in all three cell types assayed and speculate that compensatory endogenous anti-inflammatory signaling may be driving this observation, though we cannot substantiate this claim with the genes measured in this study.

The $176 \mathrm{hr}$ wd time point is meant to measure long term changes in gene expression that occur in protracted withdrawal. At this time point, some similarities across cell types were observed in the subphenotypes that highly expressed GABAR subunits as was observed at other time points. NE neuron cluster 5, GLP-1 neuron cluster 2 and microglia cluster 2 contained the majority of the GABAR subunit genes, and the makeup of this 'GABAR' co-expression cluster was not as consistent as the inflammatory cluster across cell types -16 genes are shared across all cell types and 16 genes are unique to a single cell type within its respective GABAR cluster (Sup. Table 4). GLP-1 neurons in subphenotype B upregulates this co-expression cluster in the control treatment, but the relative level of expression of this 
cluster decreases throughout the time series within this subphenotype (Fig. 4). At the $176 \mathrm{hr}$ wd time point, this GABAR cluster is only moderately expressed suggesting long term changes to this neuronal subphenotype as a result of alcohol dependence and withdrawal. The decrease in expression of inhibitory GABAR gene transcripts, along with the concurrent upregulation of co-expression cluster 3 , which contains $G c g$, suggests that this GLP-1 neuronal subphenotype experiences long term functional changes such that its neurotransmission increases. Literature indicates that GLP-1 signaling from the NTS to the amygdala and other nuclei is anxiogenic ${ }^{29}$. Taken together, these data suggest that this GLP-1 neuronal subphenotype is not primarily involved the acute withdrawal process characterized by inflammation, but rather experiences GABAR subunit downregulation over a longer process potentially leading to increased anxiety and susceptibility to stress in protracted AWS.

Microglia also showed elevated GABAR expression at the $176 \mathrm{hr}$ wd time point, but the pattern of increased GABAR expression was unexpected. Control microglia in subphenotype $\mathrm{C}$ show moderate expression of both cluster 1 (inflammatory) and cluster $2\left(G_{A B A} R\right)$ (Fig. 5). Expression of both of these clusters increases at the $176 \mathrm{hr}$ wd time point. This suggests elevated inflammation, but not by distinct M1 phenotype microglia (subphenotype A), and also elevated GABAR expression. These findings are best visualized in Fig. 8. Of note, there many genes in microglia cluster 2 that are not GABAR subunits. Moreover, microglial Tnf expression was significantly elevated in control, EtOH and $8 \mathrm{hr}$ wd treatments compared to $176 \mathrm{hr}$ wd independent of subphenotype (Sup. Table 3). Indeed, Tnf expression by microglia did not fit neatly into a gene cluster. Cluster $\mathrm{C}$ has some cells that demonstrate high thf in both control and $176 \mathrm{hr}$ wd, where Cluster A showed a decrease in Tnf expression between these two time points. Cluster B, conversely, increased its expression of Tnf from control to $176 \mathrm{hr}$ wd. This apparent absence of a pattern in microglia Tnf expression suggests that in microglia this gene that is central to neuroinflammation is constrained by a mechanism that is independent of other gene expression regulation. Further, the decrease in overall microglia Tnf expression at $176 \mathrm{hr}$ wd as measured by an average of $-\Delta \Delta C_{t}$ values and two-tailed heteroscedastic t-tests may be misleading. A single-cell analysis reveals that overall expression may not be the best indicator of inflammation. Rather, shifts in subphenotype proportion, and the number of cells showing a moderately increased Tnf expression, as seen in subphenotype $\mathrm{C}$, may have more of a physiologic impact than total gene expression levels.

Cell diagrams in Figs. 6,7,8 average the expression of a gene within a subphenotype designated by color and display that color in a location on the cell cartoon that corresponds to the protein function. This method of data presentation allows for analysis of receptor-ligand interactions within and between subphenotypes. For example, Fig. 6 suggests that subphenotype $C$ experiences an increase in both CCLCCR and CXCL10-CXCR signaling at $32 \mathrm{hr}$ wd as the ligand and receptor genes for these proteins are highly expressed. Figure 6 also provides clarity in subphenotype D upregulation of Mapk1 at $176 \mathrm{hr}$ wd suggesting long term transcription is altered during protracted withdrawal in this subphenotype. Moreover, transcription factor genes $c F o s$, Junb, $N f k B$, and Stat3 have increased expression in subphenotype D2 suggesting this subset of NE neurons undergoes substantial long-term changes in transcription following alcohol withdrawal. Microglia in subphenotype $C$ upregulate $I L 1 a, I L 1 b$, and $I L 1 r 1$ 
at $176 \mathrm{hr}$ wd in subphenotype $\mathrm{C}$ as compared to control, while subphenotype $\mathrm{B}$ downregulate these genes at $176 \mathrm{hr}$ wd compared to control (Fig. 8). This dynamic provides indirect evidence that subphenotype $B$ provides an anti-inflammatory function that is most active in protractracted withdrawal. Moreover, it suggests that microglia subphenotype $\mathrm{C}$, identified here as a microglia subset that can function in a multitude of processes whether inflammatory or anti-inflammatory based on their lack of a clear coexpression module pattern in control, is pushed towards an inflammatory state in protracted withdrawal.

This dataset has allowed the identification of cellular subphenotypes and their gene expression dynamics in alcohol withdrawal through time. Analysis has revealed valuable insights in both neurotransmission signaling and local paracrine signaling processes that relate to what is observed clinically in the context of what is already established about such neurotransmission. The dataset is unique in that microfluid RTqPCR, a method lower in throughput but more reliable than RNA-seq ${ }^{46}$, is combined with anatomic and staining specificity using LCM for single-cell selection in a time series. This allows for analysis of complex signaling dynamics at multiple levels, and the influence of such signaling dynamics on both acute AWS and protracted withdrawal based on the clinical symptoms at that time point.

We have collected the data, validated the accuracy of the dataset, and identified cellular subphenotypes and their major signaling dynamics. However, signaling dynamics measured in our dataset can be further investigated and may identify clinical targets to treat acute or protracted AWS and potentially alcohol dependence itself. Future studies analyzing these signaling dynamics with the addition of female rats that also include other brain cell types such as astrocytes and endothelial cells are needed to further understand the underlying pathophysiology of AWS and dependence.

Lastly, these findings are consistent with the hypothesis that neuroinflammation in the visceral-emotional neuraxis contributes to antireward which motivates alcohol, and opioid, dependence (Fig. 1A-B; Sup. Figure 7) ${ }^{6}$. In brief, this hypothesis suggests that neuroinflammation in the NTS and amygdala stimulates antireward which contributes to negative reinforcement. This study does not only provides evidence of neuroinflammation in the NTS in acute and protracted alcohol withdrawal, but also an understanding of the emergence of this neuroinflammation and its relation to neurotransmission and AWS. Improved understanding of such processes in alcohol withdrawal lends insights into targets that may mitigate inflammation, decrease antireward in AWS, and treat substance dependence.

\section{Methods}

\section{Animals}

Approval of protocols was given by Institutional Animal Care and Use Committee of Thomas Jefferson University. The study was carried out in compliance with ARRIVE guidelines and in accordance with all relevant guidelines and regulations. Ten young, male, Sprague Dawley rats (35-45 grams) ordered from Harlan Laboratory were housed individually in the Thomas Jefferson University Alcohol Research Center Animal Core Facility. Standard chow and water was given until rats weighed 120 grams. Rats were then 
fed an alcohol-free, maltose-dextrin substituted, Lieber DeCarli liquid diet (bioServe, Frenchtown, NJ) for three days ${ }^{47}$. Animals were then assigned to five treatment groups: control, chronic alcohol exposure (EtOH), 8 hour (hr) withdrawal (wd), $32 \mathrm{hr} w d$, or $176 \mathrm{hr}$ wd (Sup. Fig. 1). Animals received eight months of ethanol or control diet. Control diet animals received a quantity of the liquid diet that equaled the caloric intake of the matched alcohol-fed animal 24-hours prior. Withdrawal animals were withdrawn such that sacrifice by rapid decapitation was at the same circadian time for all conditions. Single-cell gene expression variance within an animal demonstrated similar or greater variance as that between animals (Sup. Fig. 2).

\section{Rapid decapitation, fast staining protocol, LCM}

Dissected brainstems were frozen in Optimal Cutting Temperature (O.C.T.) following rapid decapitation for cryostat sectioning and stored at $-80^{\circ} \mathrm{C}$ for nucleic acid preservation. An in-house rapid immunofluorescent staining protocol developed to preserve nucleic acid integrity was used to visualize cell types for single-cell laser capture microdissection (LCM) as explained elsewhere (Sup. Fig. 1B) ${ }^{38}$. Briefly, $10 \mu \mathrm{m}$ thick brain sections were thaw-mounted onto glass slides. $30 \mathrm{~s}$ of $75 \%$ ethanol fixed sliced tissue. $30 \mathrm{~s}$ of $2 \%$ BSA (Sigma-Aldrich) in phosphate-buffered saline (PBS) was used for blocking. Tissue was then stained with $3 \%$ of anti-NeuN antibody (EMD Millipore, MAB377) or anti-Cd11 $\beta$ antibody (Genway Biotech, CCEC48) for neuron and microglia primary labeling, respectively. $3 \%$ anti-tyrosine hydroxylase (Th) antibody (Abcam, ab112) was also used on NeuN stained slides for noradrenergic neuron labeling. Secondary antibodies (ratio 1:200) goat anti-mouse Alexa Fluor-555 and donkey antirabbit Alexa Fluor-488 were used for cell type and Th fluorescence, respectively. DAPI (1:10000) stained cell nuclei. PBS wash ensued, along with a standard alcohol dehydration protocol (30 s 75\% ethanol, $30 \mathrm{~s}$ $95 \%$ ethanol, $30 \mathrm{~s} 100 \%$ ethanol, $30 \mathrm{~s} 100 \%$ ethanol, $60 \mathrm{~s}$ xylenes, $4 \mathrm{~m}$ xylenes) and $5 \mathrm{~m}$ in desiccator before LCM.

\section{Single cell sampling and High-Throughput qRT-PCT}

3230 single brain cells, 950 Th+ neurons, 1030 Th- neurons, and 1250 microglia, were collected from the NTS using LCM. Cells were grouped into 10 -cell pools comprising 323 total samples analyzed. This pooling of cells increases the number of samples analyzed by the microfluidic RT-qPCR platform. cDNA from mRNA transcripts was generated by reverse transcription (SuperScript ${ }^{T M}$ VILO ${ }^{T M}$ cDNA Synthesis Kit; ThermoFisher). TaqMan PreAmp Master Mix was used for pre-amplification of cDNA (22 cycles) with forward and reverse PCR primers (96 pairs). The Biomark microfluidic qPCR platform (Fluidigm@) was used to measure expression levels of 96 genes. Four batches of probe-based qPCR measured the previously amplified 96 cDNA transcripts. Supplemental Table 1 lists primers used. Primer amplicon validation was performed on agarose gel electrophoresis. Following strict quality control protocols, a total of 229 10-cell pooled samples (70 NE neuron samples, 65 GLP-1 neuron samples, and 94 microglial samples) and 65 gene transcripts were used for data analysis. 
The four microfluidic RT-qPCR batches run for this study were assessed for intra- and inter-batch experimental quality (Sup. Fig. 3-4). Technical replicates assessing intra-batch quality demonstrated high similarity with $r$ values listed (Sup. Fig. 3). Inter-batch replicates demonstrated high batch similarity, though batch 4 sample 40 showed contamination (Sup. Fig. 4). A dilution series using standard rat brain RNA was also included in each batch for quantitative analysis (Sup. Table 2); However, the data normalization method explained below calculated relative expression and was used for all analysis in this study.

\section{Data Normalization}

A two-step median-centering - $\Delta \Delta \mathrm{C}_{\mathrm{t}}$ method was used for expression level normalization was explained elsewhere ${ }^{48}$. Briefly, a raw $C_{t}$ value was obtained for each gene and sample. Each individual $C_{t}$ value was normalized to the overall sample median ((Median sample expression) $-C_{t \text { gene }}=-\Delta C_{t \text { sample }}$ ). The newly obtained $-\Delta C_{t}$ values were then median-centered to the gene across all samples $\left(-\Delta C_{t}\right.$ sample - (Across sample $-\Delta \mathrm{C}_{\mathrm{t}}$ median $)=-\Delta \Delta \mathrm{C}_{\mathrm{t} \text { gene }}$ ). This yields a $-\Delta \Delta \mathrm{C}_{\mathrm{t}}$ value for each measurement allowing comparison of relative gene expression values across treatment groups and batches. This analysis was carried out in $R$ version 3.5.2. The raw $C_{t}$ values are listed in Supplemental Table 2 without gene quality control. The normalized dataset with quality-control that was used for all analysis is also displayed in Supplemental Table 2.

\section{Declarations}

\section{Acknowledgments}

SJO would like to acknowledge Dr. Jan Hoek for his support with the T32 AA 007463.

\section{Author Contributions}

SJO performed microfluidic qPCR, data analysis, figure generation, and writing of manuscript. DMC collected single-cell samples under the guidance of JP. JP also designed the experiments. RV and JSS were involved with figure design and editing. All authors discussed the results and commented on the manuscript.

\section{Competing Interests Statement}

The authors declare that the research was conducted in the absence of any commercial, financial, or nonfinancial relationships that could be construed as competing interests.

\section{References}


1. Kosten, T. R. \& O'Connor, P. G. Management of Drug and Alcohol Withdrawal. N. Engl. J. Med. 348, 1786-1795 (2003).

2. Baker, T. B., Piper, M. E., McCarthy, D. E., Majeskie, M. R. \& Fiore, M. C. Addiction Motivation Reformulated: An Affective Processing Model of Negative Reinforcement. Psychol. Rev. 111, 33-51 (2004).

3. Retson, T. A., Sterling, R. C. \& Van Bockstaele, E. J. Alcohol-induced dysregulation of stress-related circuitry: The search for novel targets and implications for interventions across the sexes. Prog. Neuropsychopharmacol. Biol. Psychiatry (2015) doi:10.1016/j.pnpbp.2015.05.009.

4. Koob, G. F. \& Le Moal, M. Drug Addiction, Dysregulation of Reward, and Allostasis. Neuropsychopharmacology 24, 97-129 (2001).

5. Koob, G. F. \& Le Moal, M. Review. Neurobiological mechanisms for opponent motivational processes in addiction. Philos. Trans. R. Soc. Lond. B. Biol. Sci. 363, 3113-3123 (2008).

6. O'Sullivan, S. J. \& Schwaber, J. S. Similarities in alcohol and opioid withdrawal syndromes suggest common negative reinforcement mechanisms involving the interoceptive antireward pathway. Neurosci. Biobehav. Rev. 125, 355-364 (2021).

7. Koob, G. F. Brain stress systems in the amygdala and addiction. Brain Res. 1293, 61-75 (2009).

8. McBride, W. J. et al. Changes in gene expression in regions of the extended amygdala of alcoholpreferring rats after binge-like alcohol drinking. Alcohol 44, 171-183 (2010).

9. Freeman, K. et al. Temporal changes in innate immune signals in a rat model of alcohol withdrawal in emotional and cardiorespiratory homeostatic nuclei. J. Neuroinflammation 9, 97 (2012).

10. Freeman, K. et al. Coordinated dynamic gene expression changes in the central nucleus of the amygdala during alcohol withdrawal. Alcohol. Clin. Exp. Res. 37 Suppl 1, E88-100 (2013).

11. Whitman, B. A., Knapp, D. J., Werner, D. F., Crews, F. T. \& Breese, G. R. The Cytokine mRNA Increase Induced by Withdrawal from Chronic Ethanol in the Sterile Environment of Brain is Mediated by CRF and HMGB1 Release. Alcohol. Clin. Exp. Res. 37, 2086-2097 (2013).

12. Breese, G. R. \& Knapp, D. J. Persistent adaptation by chronic alcohol is facilitated by neuroimmune activation linked to stress and CRF. Alcohol 52, 9-23 (2016).

13. O'Sullivan, S. J. et al. Single-Cell Glia and Neuron Gene Expression in the Central Amygdala in Opioid Withdrawal Suggests Inflammation With Correlated Gut Dysbiosis. Front. Neurosci. 13, 665 (2019).

14. Roberto, M., Kirson, D. \& Khom, S. The Role of the Central Amygdala in Alcohol Dependence. Cold Spring Harb. Perspect. Med. a039339 (2020) doi:10.1101/cshperspect.a039339.

15. Phelps, E. A. \& LeDoux, J. E. Contributions of the Amygdala to Emotion Processing: From Animal Models to Human Behavior. Neuron 48, 175-187 (2005).

16. Yang, L. et al. Systemic inflammation induces anxiety disorder through CXCL12/CXCR4 pathway. Brain. Behav. Immun. 56, 352-362 (2016).

17. Schäfers, M. \& Sorkin, L. Effect of cytokines on neuronal excitability. Neurosci. Lett. 437, 188-193 (2008). 
18. Covarrubias, M. Y., Khan, R. L., Vadigepalli, R., Hoek, J. B. \& Schwaber, J. S. Chronic alcohol exposure alters transcription broadly in a key integrative brain nucleus for homeostasis: the nucleus tractus solitarius. Physiol. Genomics 24, 45-58 (2005).

19. McDonald, M., Hoek, J., Ogunnaike, B. \& Schwaber, J. Behavioral and neurobiological changes within a period of heightened susceptibility to voluntary alcohol withdrawal. FASEB J. 22, 946.7-946.7 (2008).

20. Bär, K. J. et al. Reduced baroreflex sensitivity in acute alcohol withdrawal syndrome and in abstained alcoholics. Drug Alcohol Depend. 85, 66-74 (2006).

21. King, A. C., Errico, A. L., Parsons, O. A. \& Lovallo, W. R. Blood Pressure Dysregulation Associated with Alcohol Withdrawal. Alcohol. Clin. Exp. Res. 15, 478-482 (1991).

22. Herman, J. P. Neural pathways of stress integration relevance to alcohol abuse. Alcohol Research: Current Reviews vol. 34 441-447 (2012).

23. Holt, M. K. \& Trapp, S. The physiological role of the brain GLP-1 system in stress. Cogent Biol. 2, 1229086 (2016).

24. Maniscalco, J. W. \& Rinaman, L. Vagal Interoceptive Modulation of Motivated Behavior. Physiology 33, 151-167 (2018).

25. Han, V. K. M. et al. Cellular localization of proglucagon/glucagon-like peptide I messenger RNAs in rat brain. J. Neurosci. Res. 16, 97-107 (1986).

26. Larsen, P. J., Tang-Christensen, M., Holst, J. J. \& Orskov, C. Distribution of glucagon-like peptide-1 and other preproglucagon-derived peptides in the rat hypothalamus and brainstem. Neuroscience 77, 257-70 (1997).

27. Gu, G. et al. Glucagon-like peptide-1 in the rat brain: distribution of expression and functional implication. J. Comp. Neurol. 521, 2235-2261 (2013).

28. Zheng, H., Cai, L. \& Rinaman, L. Distribution of glucagon-like peptide 1-immunopositive neurons in human caudal medulla. Brain Struct. Funct. 220, 1213-9 (2015).

29. Rinaman, L. Interoceptive stress activates glucagon-like peptide-1 neurons that project to the hypothalamus. Am. J. Physiol. 277, R582-90 (1999).

30. Vallöf, D., Vestlund, J. \& Jerlhag, E. Glucagon-like peptide-1 receptors within the nucleus of the solitary tract regulate alcohol-mediated behaviors in rodents. Neuropharmacology 149, 124-132 (2019).

31. Kalia, M., Fuxe, K. \& Goldstein, M. Rat medulla oblongata. II. Dopaminergic, noradrenergic (A1 and A2) and adrenergic neurons, nerve fibers, and presumptive terminal processes. J. Comp. Neurol. 233, 308-332 (1985).

32. Ferry, B., Roozendaal, B. \& McGaugh, J. L. Role of norepinephrine in mediating stress hormone regulation of long-term memory storage: a critical involvement of the amygdala. Biol. Psychiatry 46, 1140-1152 (1999). 
33. Williams, C. L., Men, D., Clayton, E. C. \& Gold, P. E. Norepinephrine release in the amygdala after systemic injection of epinephrine or escapable footshock: contribution of the nucleus of the solitary tract. Behav. Neurosci. 112, 1414-22 (1998).

34. DeCicco, D., Zhu, H., Brureau, A., Schwaber, J. S. \& Vadigepalli, R. MicroRNA network changes in the brain stem underlie the development of hypertension. Physiol. Genomics 47, 388-399 (2015).

35. Waki, H., Gouraud, S. S., Maeda, M. \& Paton, J. F. R. Evidence of specific inflammatory condition in nucleus tractus solitarii of spontaneously hypertensive rats. Exp. Physiol. 95, 595-600 (2010).

36. Ray, L. A. et al. Development of the Neuroimmune Modulator Ibudilast for the Treatment of Alcoholism: A Randomized, Placebo-Controlled, Human Laboratory Trial. Neuropsychopharmacology 42, 1776-1788 (2017).

37. Park, J. et al. Inputs drive cell phenotype variability. Genome Res. 24, 930-941 (2014).

38. Park, J. et al. Single-Cell Transcriptional Analysis Reveals Novel Neuronal Phenotypes and Interaction Networks Involved in the Central Circadian Clock. Front. Neurosci. 10, 481 (2016).

39. O'Sullivan, S. J., Reyes, B. A. S., Vadigepalli, R., Van Bockstaele, E. J. \& Schwaber, J. S. Combining laser capture microdissection and microfluidic qpcr to analyze transcriptional profiles of single cells: A systems biology approach to opioid dependence. J. Vis. Exp. 2020, e60612 (2020).

40. Murray, P. J. et al. Macrophage Activation and Polarization: Nomenclature and Experimental Guidelines. Immunity 41, 14-20 (2014).

41. Wolf, Y., Yona, S., Kim, K. W. \& Jung, S. Microglia, seen from the CX3CR1 angle. Front. Cell. Neurosci. 7. (2013).

42. Becker, H. C. Animal models of alcohol withdrawal. Alcohol Res. Health 24, 105-13 (2000).

43. Martinez, F. O. \& Gordon, S. The M1 and M2 paradigm of macrophage activation: time for reassessment. F1000Prime Rep. 6, 13 (2014).

44. Freeman, K. et al. Coordinated Dynamic Gene Expression Changes in the Central Nucleus of the Amygdala During Alcohol Withdrawal. Alcohol. Clin. Exp. Res. 37, E88-100 (2013).

45. Freeman, K. et al. Rapid temporal changes in the expression of a set of neuromodulatory genes during alcohol withdrawal in the dorsal vagal complex: molecular evidence of homeostatic disturbance. Alcohol. Clin. Exp. Res. 36, 1688-1700 (2012).

46. Consortium, S.-I. et al. A comprehensive assessment of RNA-seq accuracy, reproducibility and information content by the Sequencing Quality Control Consortium. Nat. Biotechnol. 32, 903-914 (2014).

47. Lieber, C. S. \& Decarli, L. M. [58] Animal models of chronic ethanol toxicity. in 585-594 (1994). doi:10.1016/S0076-6879(94)33061-1.

48. Achanta, S. et al. Single cell gene expression analysis identifies chronic alcohol-mediated shift in hepatocyte molecular states after partial hepatectomy. Gene Expr. (2018) doi:10.3727/105221618X15361728786767. 


\section{Figures}

A

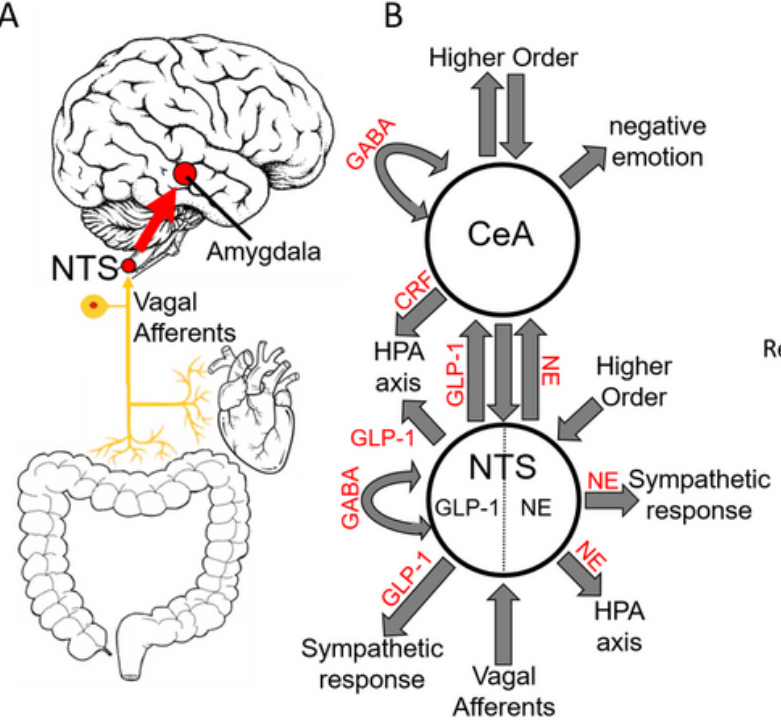

D

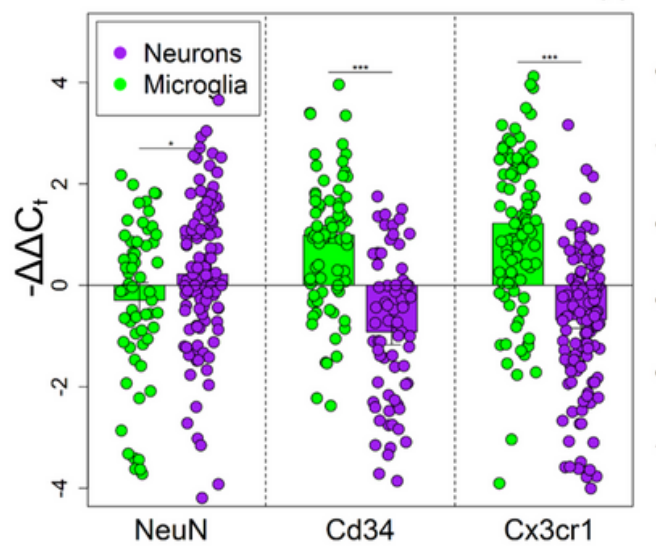

Cell Type Markers

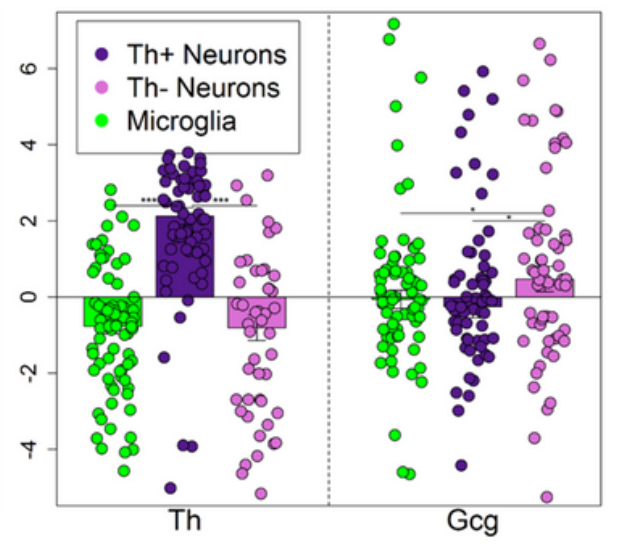

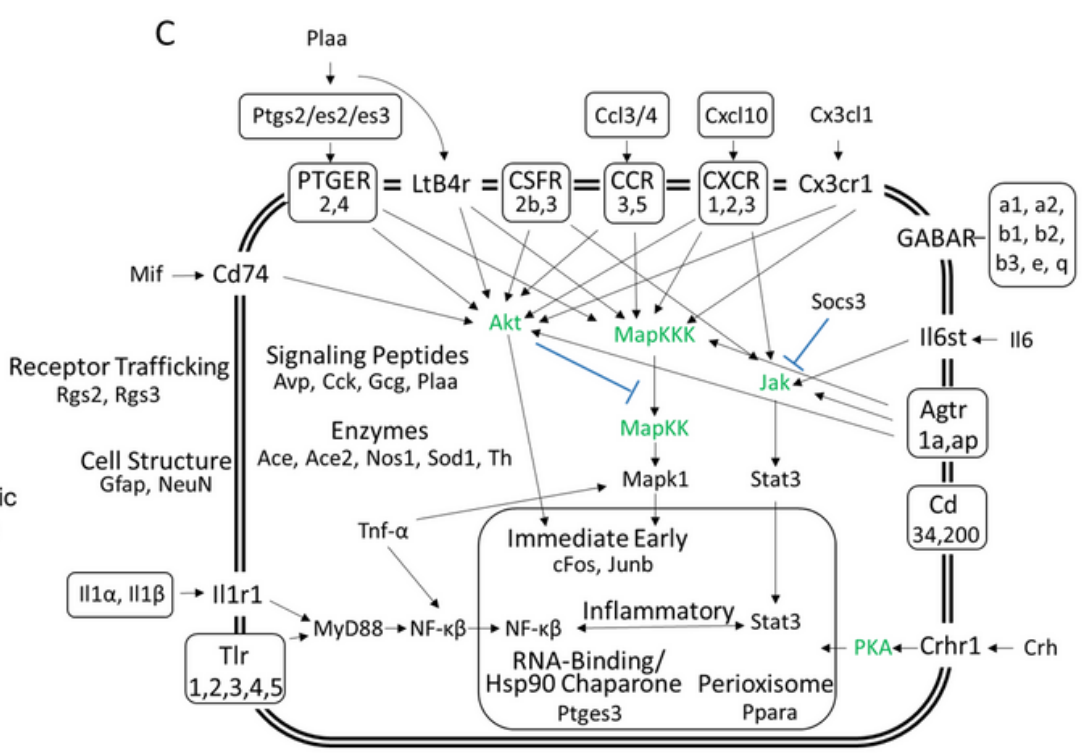

E Linear Discriminate Analysis

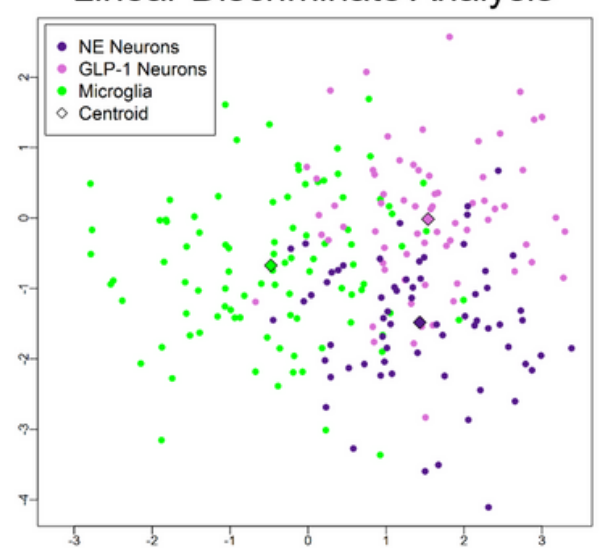

Figure 1

Experimental Design and Single-Cell Selection. (A) Rat triplets were randomly assigned one of five treatments. (B) Nucleus tractus solitarius (NTS) is primary viscerosensory nucleus of brain that relays inputs to limbic system via the central nucleus of the amygdala (CeA). Originally published in O'Sullivan et al. 2021. (C) Cartoon representation displaying NTS and CeA functions in emotion, stress, and autonomic regulation. NTS GLP-1 and NE neurons are highlighted. Many anatomical and functional connections are omitted for clarity (NE, norepinephrine; GLP-1, glucagon-like peptide 1; GABA, Yaminobutyric acid; HPA axis, hypothalamic-pituitary-adrenal axis; CRF, corticotropin releasing hormone). Originally published in O'Sullivan et al. 2021. (D) Cartoon representation of genes assayed and their function. Genes in green were not assayed. Official gene symbol is used. (E) Gene expression of cell type markers. Error bars show standard error. Neurons compared to microglia p-values $=0.0273,3.94 \mathrm{E}-10$, 7.73E-12, respectively. Th+ neurons showed elevated Th expression compared to Th- neurons $(p=4.56 \mathrm{E}-$ 11 ) and microglia $(p=2.95678 E-15)$. Th- neurons showed elevated expression of Gcg compared to Th+ neurons $(p=0.0106)$ and microglia $(p=0.0435)$ indicating they are GLP-1+ neurons. ${ }^{*} p<0.05,{ }^{* *} p<4 E-10$. 
(F) Linear discriminate analysis of all samples displays the difference across all genes measured between the three cell types collected in a two-dimension space. Centroid distance between NE neurons and GLP-1 neurons =3.30, NE neurons and microglia = 1.57, GLP-1 Neurons and microglia: 2.92.
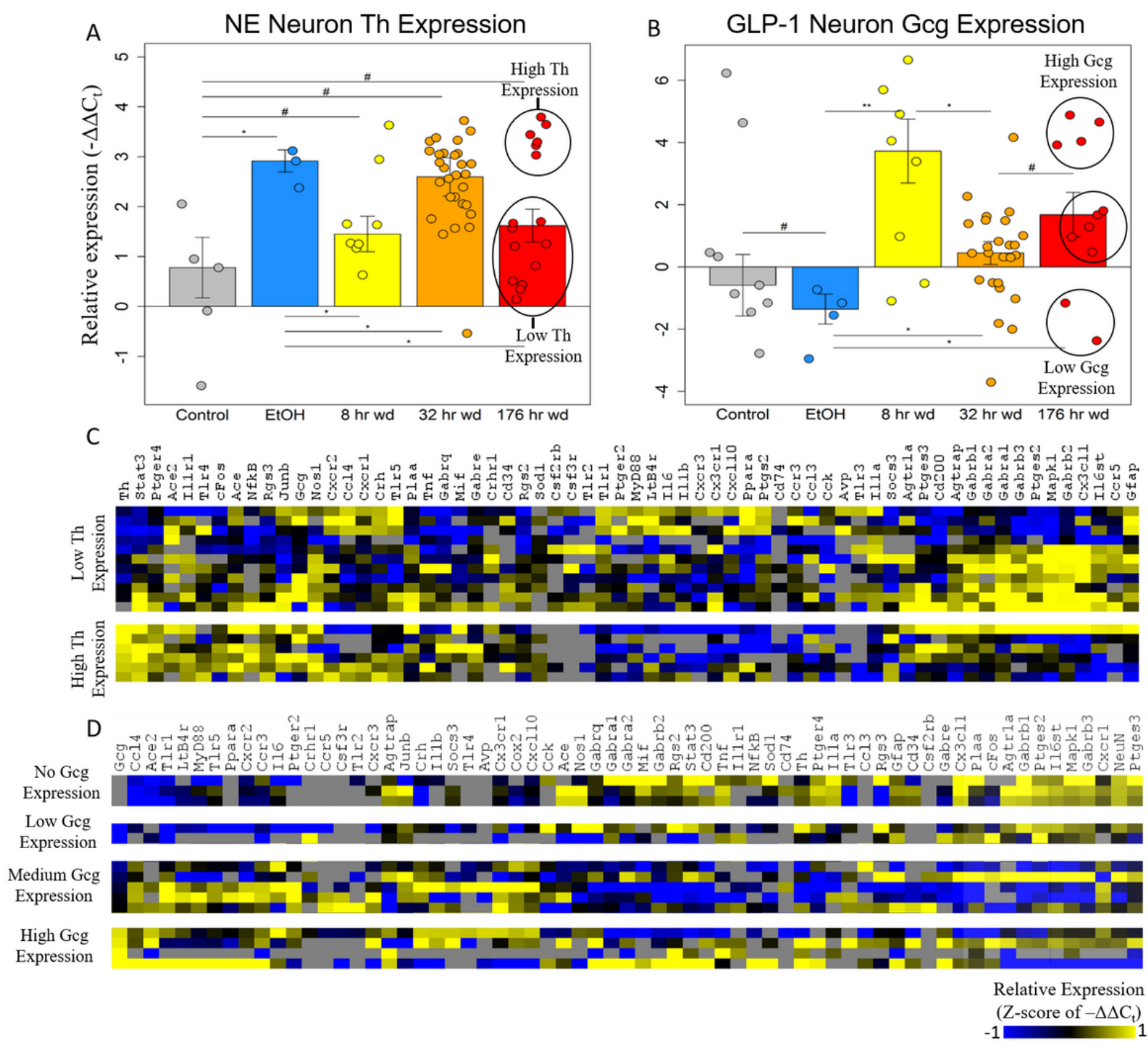

\section{Figure 2}

Neurotransmitter Expression in Neurons. ${ }^{*} p<0.003{ }^{*} p<0.05, \# p<0.1$; two-tailed heteroscedastic t-test. Bars show standard error. (A) Tyrosine hydroxylase expression (Th) in norepinephrine (NE) neurons. Bimodal Th expression at the $176 \mathrm{hr}$ wd time point is explored in the heat map in panel C. (B) Preproglucagon (Gcg) expression in GLP-1 neurons. Trimodal Gcg expression at the $176 \mathrm{hr}$ wd time point is explored in the heat map in panel D. (C) Heat map exploring high Th-expressing neurons and low Th-expressing neurons as subclusters of NE neurons in the $176 \mathrm{hr}$ wd treatment. Single-cell gene expression is shown as z-scores of $-\Delta \Delta$ Ct values. Neurotransmitter expression levels does not determine high-template co- 
expression gene clusters. (D) Heat map exploring high, middle, and low Gcg-expressing neurons as subclusters of Gcg neurons in the $176 \mathrm{hr}$ wd treatment. Single-cell gene expression is shown as z-scores of $-\Delta \Delta$ Ct values. Neurotransmitter expression levels does is more predictive of co-expression gene clusters than Th.

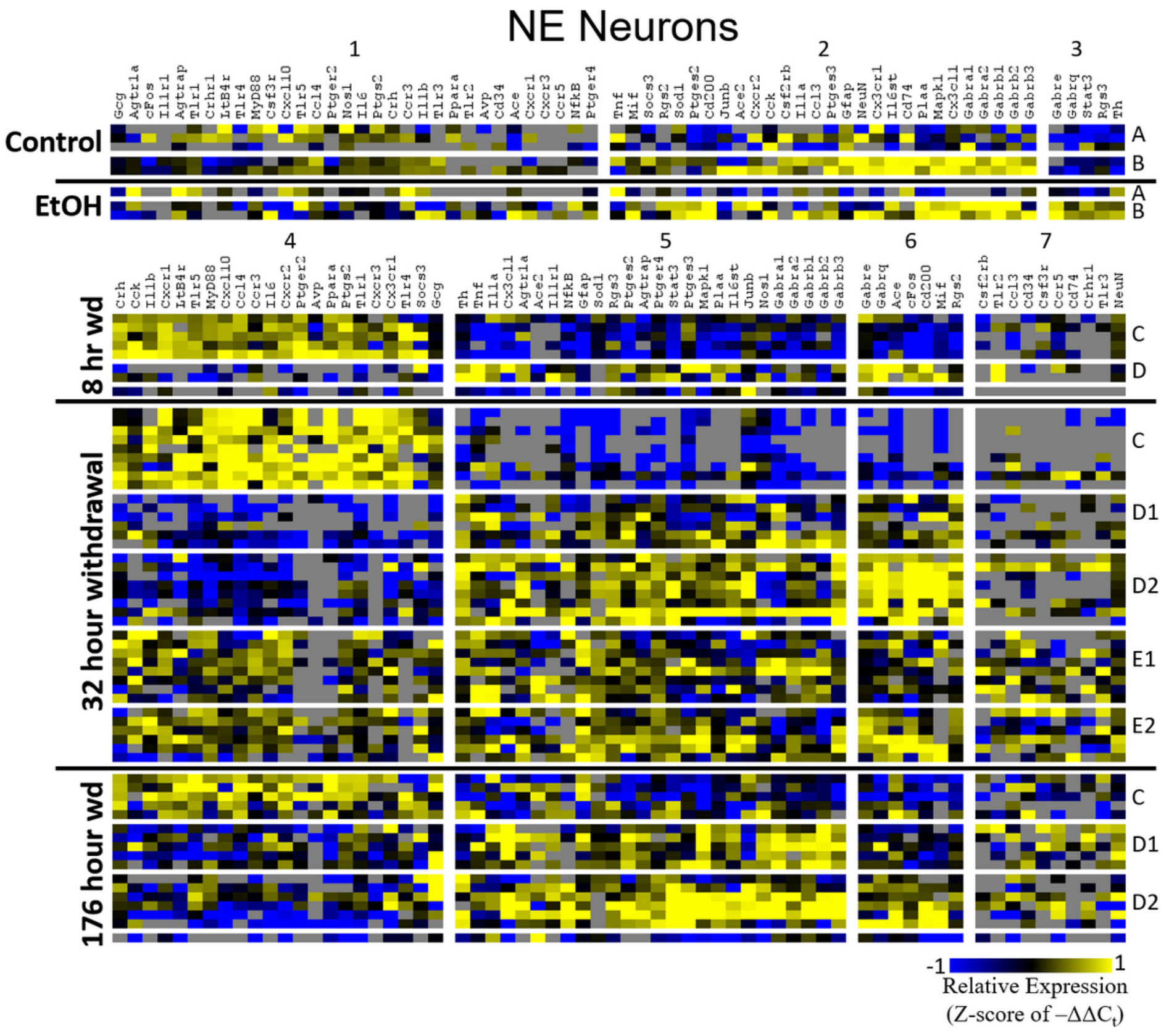

Figure 3

Heat Map of NE Neurons. Heat map displays NE neuron cellular subphenotypes through alcohol withdrawal time series. Rows represent 10-cell pooled samples with cellular subphenotype clusters labeled with uppercase letters. Columns represent the z-score of $-\Delta \Delta \mathrm{Ct}$ gene expression values on a -1 to 1 color scale for that gene in that sample. Gene clusters are labeled by number. 


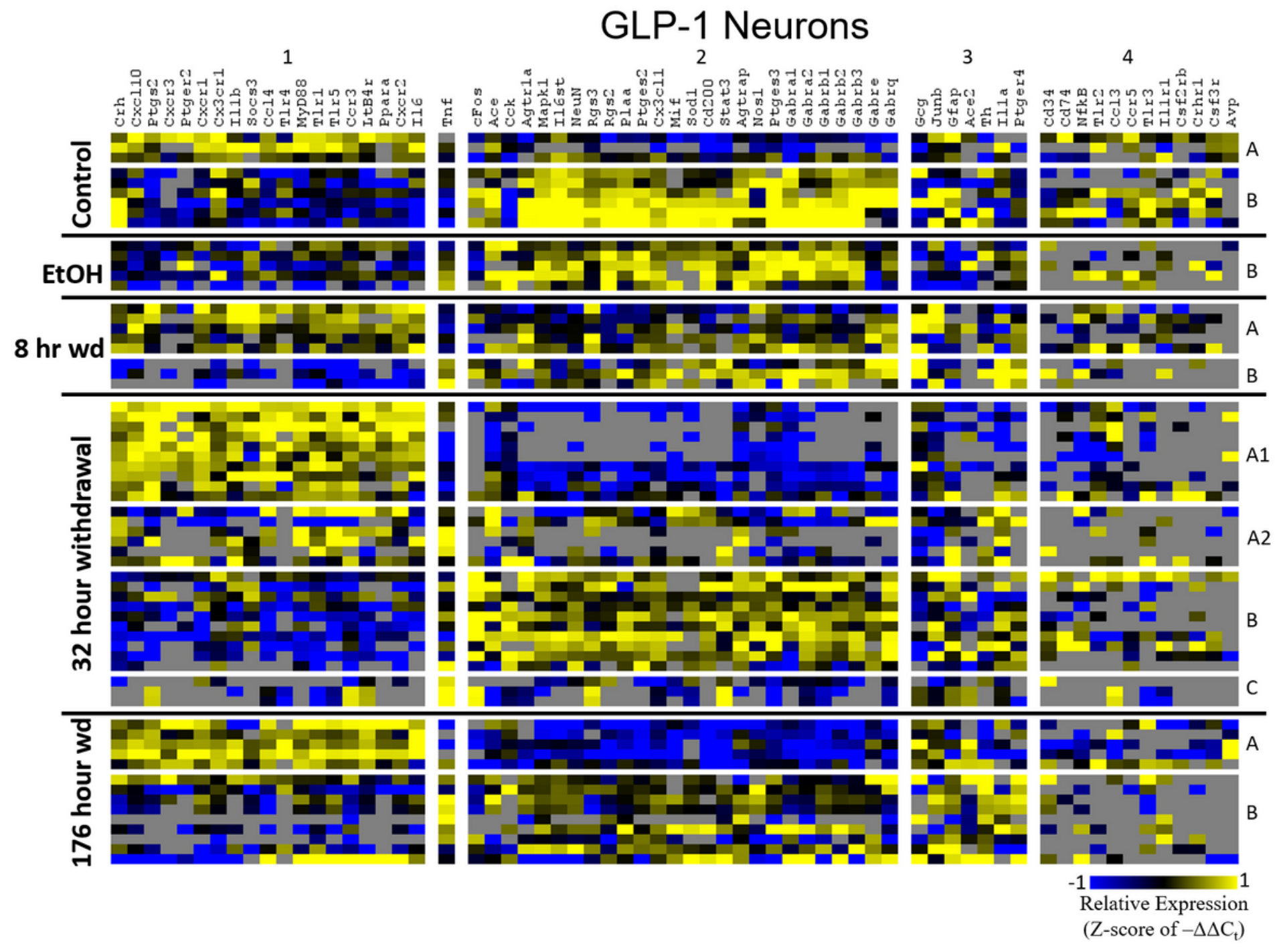

\section{Figure 4}

Heat Map of GLP-1 Neurons. Heat map displays GLP-1 neuron cellular subphenotypes through alcohol withdrawal time series. Rows represent 10-cell pooled samples with cellular subphenotype clusters labeled with uppercase letters. Columns represent the z-score of $-\Delta \Delta \mathrm{Ct}$ gene expression values on a -1 to 1 color scale for that gene in that sample. Gene clusters are labeled by number. 


\section{Microglia}

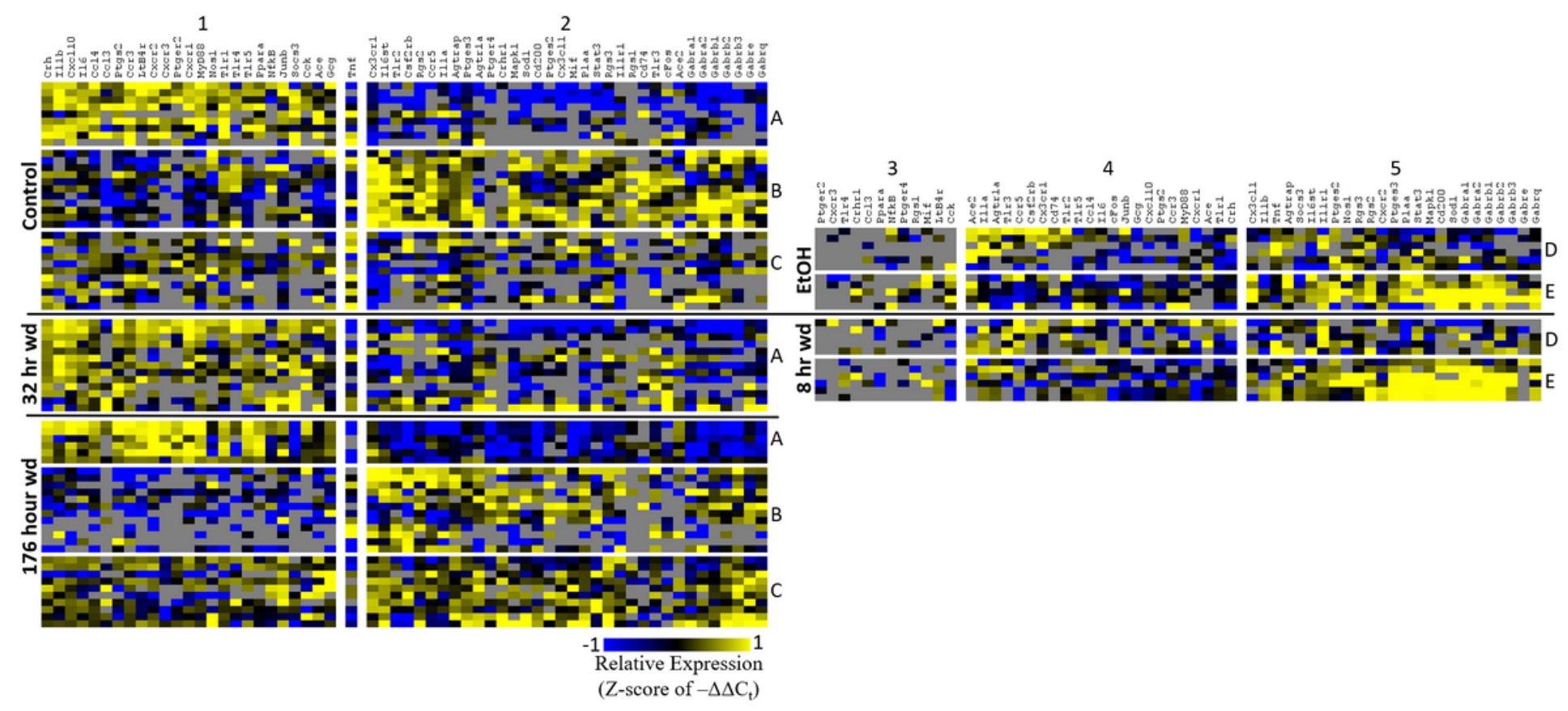

\section{Figure 5}

Heat Map of Microglia. Heat map displays microglia cellular subphenotypes through alcohol withdrawal time series. Rows represent 10-cell pooled samples with cellular subphenotype clusters labeled with uppercase letters. Columns represent the $z$-score of $-\Delta \Delta C t$ gene expression values on a -1 to 1 color scale for that gene in that sample. Gene clusters are labeled by number. 
NE Neurons

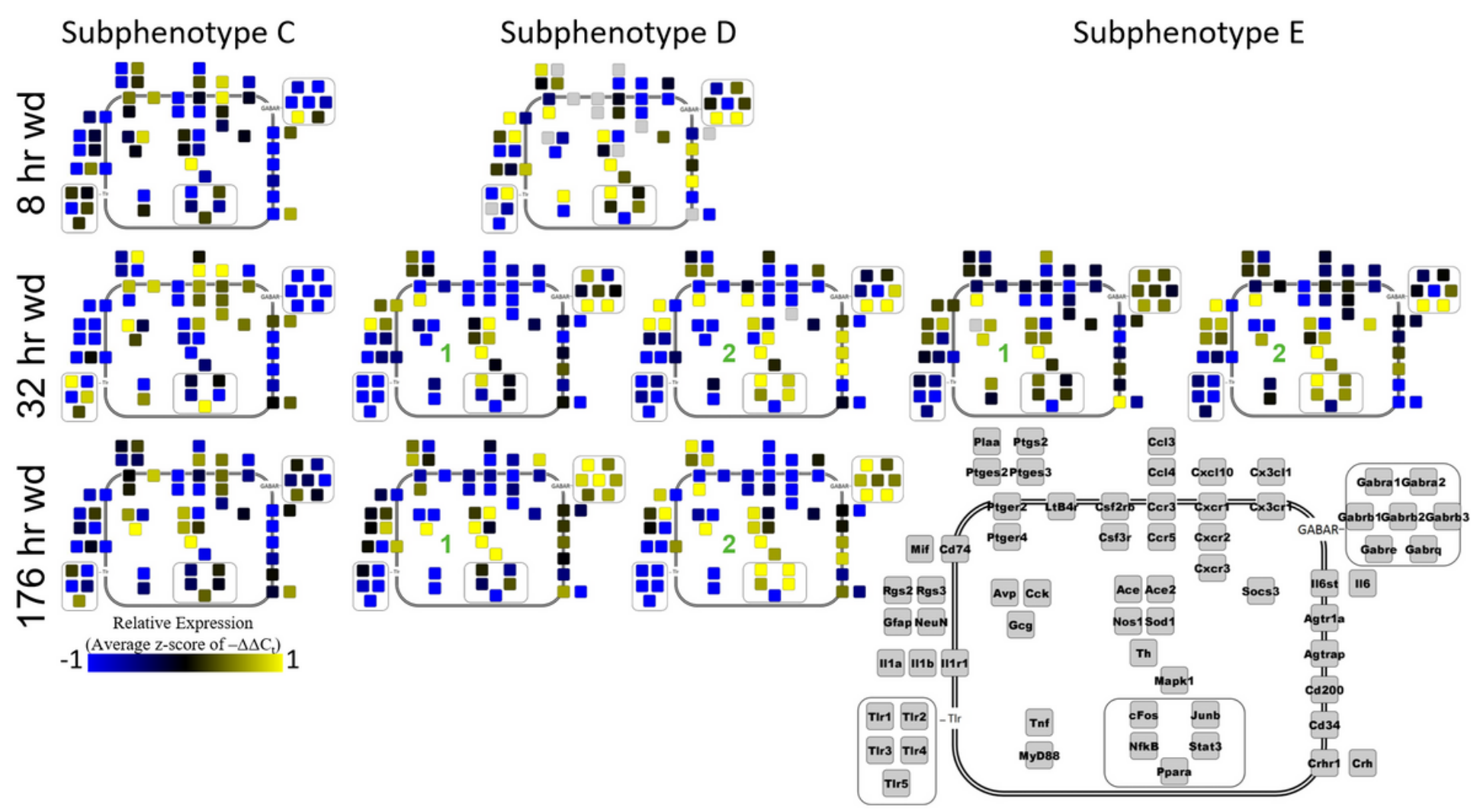

Figure 6

Suphenotype Gene Expression in Norepinephrine Neurons. Cellular diagrams display boxes representing relative gene expression (average z-score of $-\Delta \Delta$ Ct values) of subphenotypes shown in prior heatmaps. Legend with grey boxes in lower right labels which boxes correspond to which gene. Box color represents expression (blue is low expression and yellow is high expression). The location of the box represents the localization or function of the protein product from that gene transcript. Green numbers indicate subgroups within subphenotypes. Groups A and B shown in Supplemental Fig. 5. 


\section{GLP-1 Neurons}

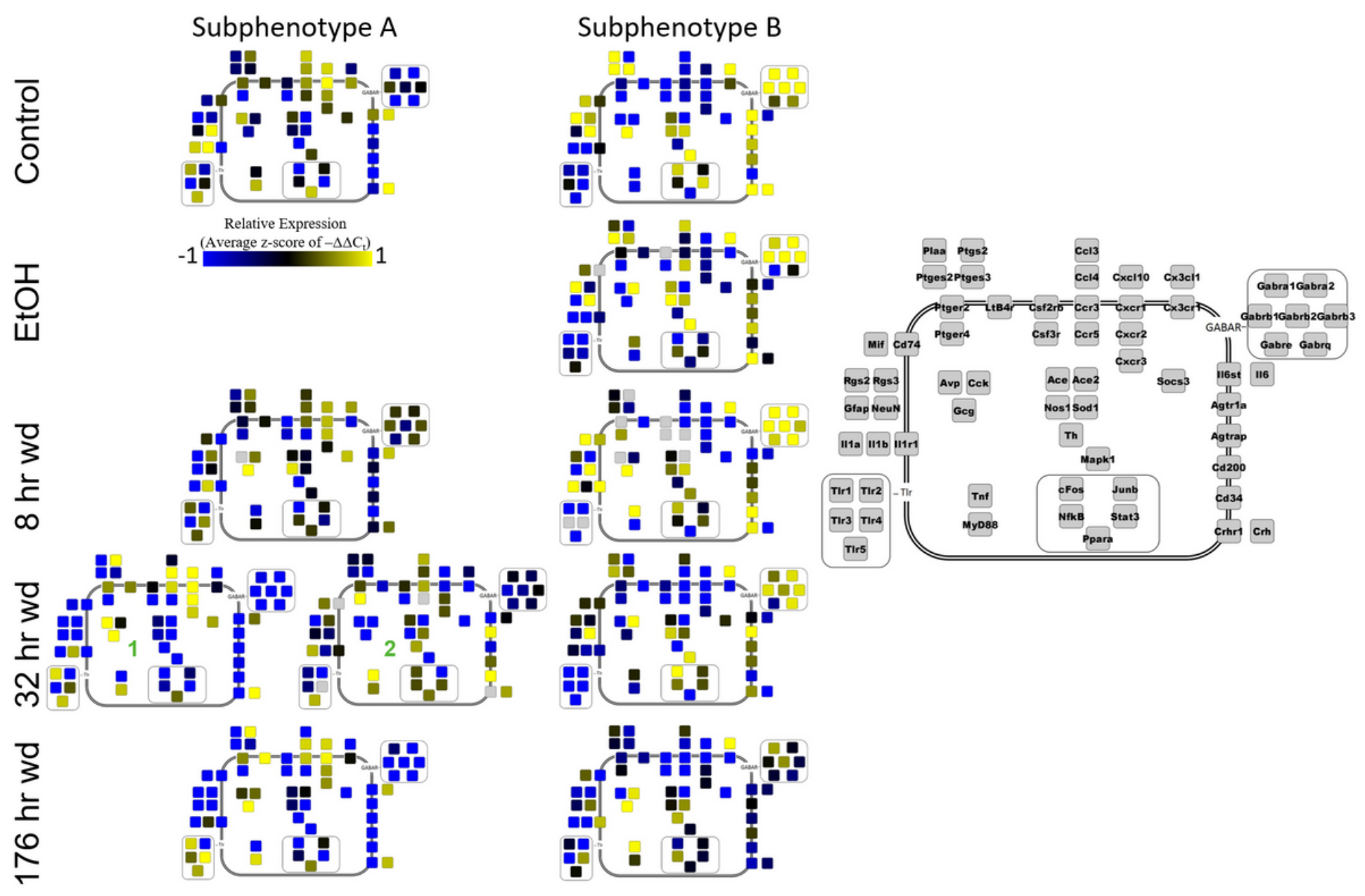

Figure 7

Suphenotype Gene Expression in GLP-1 Neurons. Cellular diagrams display boxes representing relative gene expression (average z-score of $-\Delta \Delta C t$ values) of subphenotypes shown in prior heatmaps. Legend with grey boxes in on right of figure labels which boxes correspond to which gene. Box color represents expression (blue is low expression and yellow is high expression). The location of the box represents the localization or function of the protein product from that gene transcript. Green numbers indicate subgroups within subphenotypes. Group C shown in Supplemental Fig. 5. 
Microglia

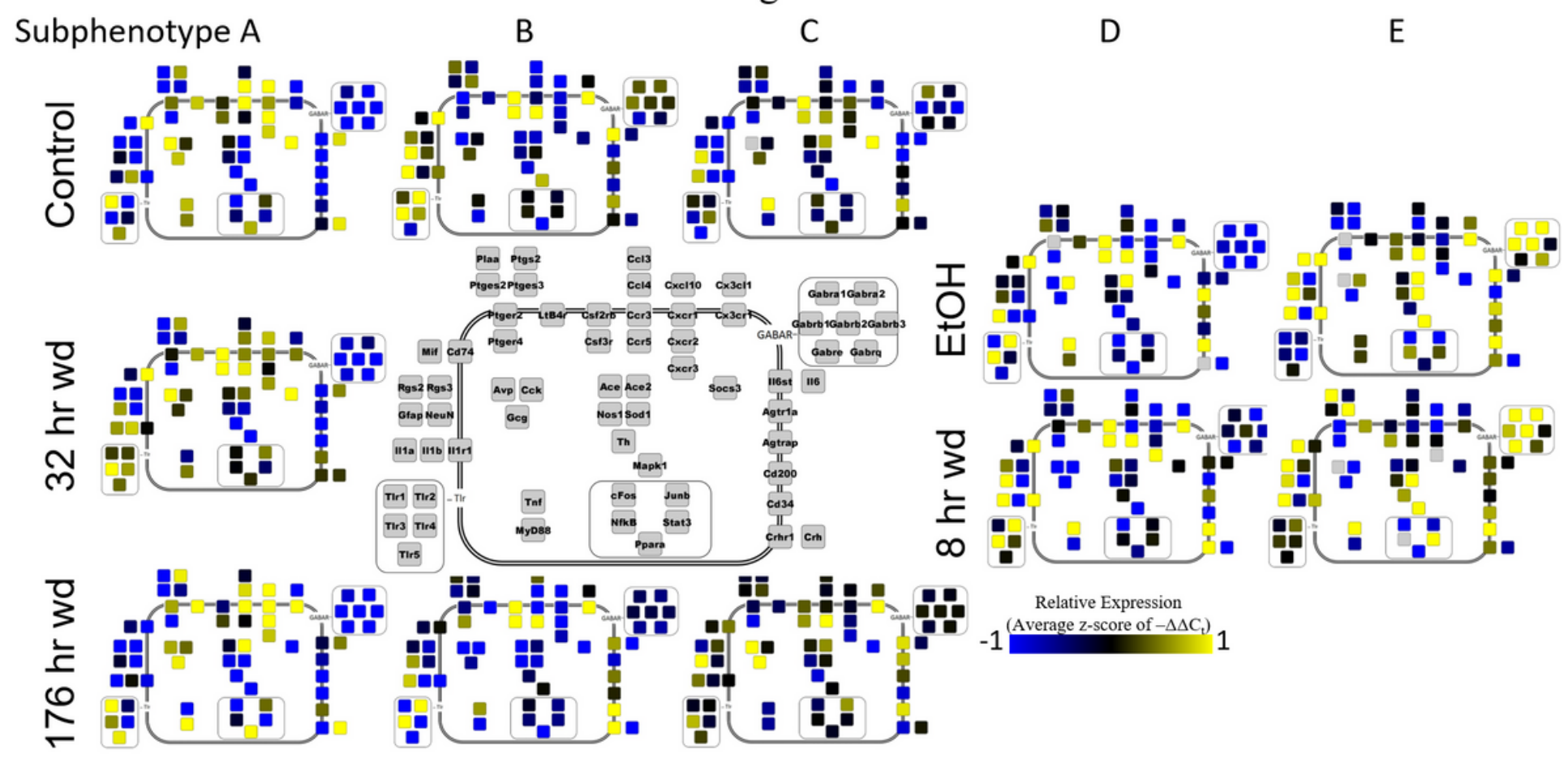

Figure 8

Suphenotype Gene Expression in Norepinephrine Neurons. Cellular diagrams display boxes representing relative gene expression (average z-score of $-\Delta \Delta$ Ct values) of subphenotypes shown in prior heatmaps. Legend with grey boxes in center of figure labels which boxes correspond to which gene. Box color represents expression (blue is low expression and yellow is high expression). The location of the box represents the localization or function of the protein product from that gene transcript.

\section{Supplementary Files}

This is a list of supplementary files associated with this preprint. Click to download.

- SupplementalFigures2.pdf

- SupplementalTable1Primers.xlsx

- SupplementalTable2Data.xlsx

- SupplementalTable3DifferentialExpression.xlsx

- SupplementalTable4HeatMapGeneclusters.xIsx 